\title{
A Silicon Micromachined Flow Sensor Using Thermopiles for Heat Transfer Measurements
}

\author{
Seiji Oda, Mitsuyoshi Anzai, Shoichi Uematsu, and Kenzo Watanabe, Fellow, IEEE
}

Abstract-A silicon micromachined flow sensor composed of a platinum heater and four thermopiles surrounding the heater is described. Two of the four thermopiles placed up and downstream in the flow measure the amount of heat carried by the fluid, while the other two arranged perpendicular to the flow direction monitor, the heat transferred from the heater to the fluid. This architecture allows the normalization of output of the up and downstream thermopiles by the monitored output. Experimental results show that the normalization is quite useful for achieving a rangeablity as wide as 1:1000 and also for reducing temperature and pressure dependence.

Index Terms-Flow sensor, heat transfer, normalization, silicon micromachining, thermopiles.

\section{INTRODUCTION}

$\mathrm{F}$ LOW meters based on heat transfer allow mass and pressure-loss-free flow measurements. Their principles of measurement can be divided into two methods: One is hot-wire anemometry, which is widely applied to omni-directional flow measurements [1]-[4]. The other is the heat propagation method [5], [6]. This method requires temperature sensors in addition to a heat source, but arranging two temperature sensors symmetrically with the heat source allows the directional measurement of laminar flow. Therefore, the propagation method facilitates measurement of accumulated flow quantity, even if the flow is pulsating. To widely apply this method in gas-meters, micromachining technologies are used to fabricate a heater and temperature sensors on a silicon chip [7]-[9]. As a temperature sensor for such a micromachined flow sensor, a fine platinum wire formed by deposition is used for easy fabrication.

The amount of heat $\mathrm{Q}$ transferred from the heat source to a fluid is empirically given by

$$
\mathrm{Q}=(\mathrm{A}+\mathrm{B} \sqrt{v})\left(\mathrm{T}_{\mathrm{h}}-\mathrm{T}_{\mathrm{f}}\right)
$$

where $\mathrm{A}$ and $\mathrm{B}$ are constants depending on thermal properties of the fluid, $v$ is fluid velocity, and $\mathrm{T}_{\mathrm{h}}$ and $\mathrm{T}_{\mathrm{f}}$ are heater and fluid temperatures, respectively [2]. To reduce temperature dependence and thereby increase rangeability, the temperature difference, $\mathrm{T}_{\mathrm{h}}-\mathrm{T}_{\mathrm{f}}$, should be kept constant and the sensor should detect the temperature change due to the heat transferred by the fluid [10]-[12]. Constant temperature-difference operation of the heater is also useful for reducing power dissipation of the micromachined sensor, but requires another sensor for detecting

Manuscript received June 15, 2002; revised April 22, 2003.

S. Oda, M. Anzai, and S. Uematsu are with the Yazaki Technology Center, Yazaki Corporation, Susono, Japan.

K. Watanabe is with the Research Institute of Electronics, Shizuoka University, Hamamatsu, Japan.

Digital Object Identifier 10.1109/TIM.2003.815997

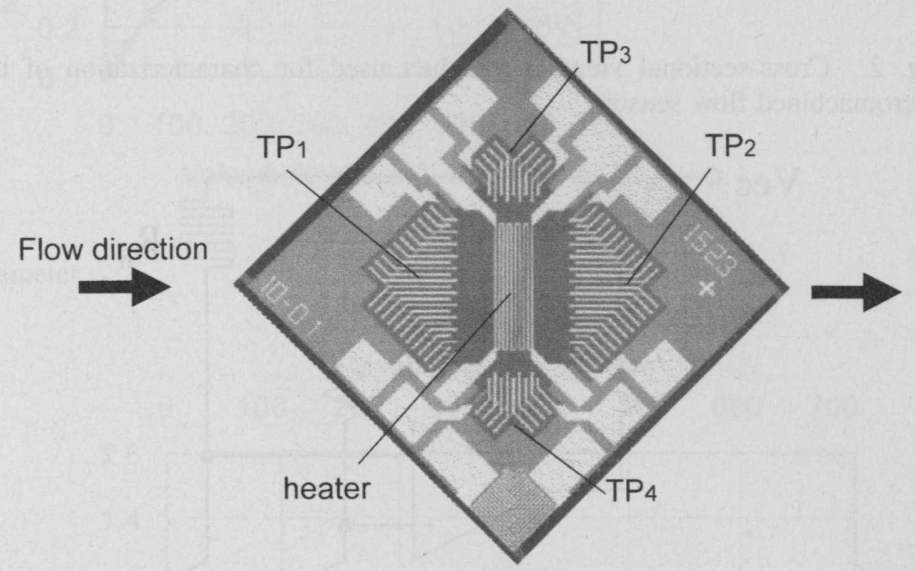

Fig. 1. Microphotograph of the micromachined flow sensor.

fluid temperature and makes for a complicated heater drive circuit. If the heat carried by the fluid is normalized by the heat transferred to the fluid, a reduction in temperature dependence is possible with a much simpler drive circuit [13]. As for the temperature sensor to measure the heat, a thermopile is superior to a platinum wire because it generates an electromotive force (EMF) proportional to the temperature difference between the hot and cold junctions. Based on these ideas, a micromachined flow sensor using four thermopiles has been developed [14], [15]. A description of its structure and performance follows.

\section{FLOW SENSOR}

Fig. 1 is a photograph of the micromachined flow sensor developed. The chip measures $2 \mathrm{~mm} \times 2 \mathrm{~mm}$ and is composed of the heater and four thermopiles surrounding it. The heater is formed by depositing platinum onto the central part of an electrically and thermally isolated silicon diaphragm. The two thermopiles, $\mathrm{TP}_{1}$ and $\mathrm{TP}_{2}$, which are arranged along the flow direction to measure the heat carried by the fluid, consist of 23 stacked thermocouples. The other two, $\mathrm{TP}_{3}$ and $\mathrm{TP}_{4}$, which are arranged perpendicular to the flow to monitor the amount of heat transferred to the fluid, consist of nine stacked thermocouples. Each thermocouple consists of platinum and highly boron-doped silicon. The hot junctions are arranged on the diaphragm and the cold junctions on the thick substrate. The substrate has high thermal conductivity and the cold junctions are assumed to stay at the fluid temperature.

\section{SENSOR PERFORMANCE}

\section{A. Experimental Conditions}

Fig. 2 shows a cross-section of the duct used to evaluate the micromachined flow sensor performance. This sensor is 


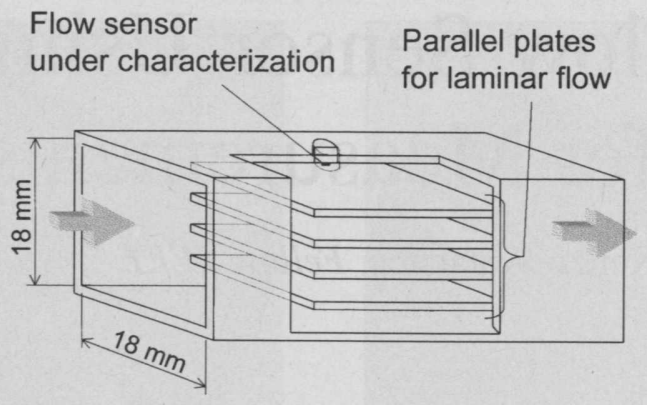

Fig. 2. Cross-sectional view of the duct used for characterization of the micromachined flow sensor.

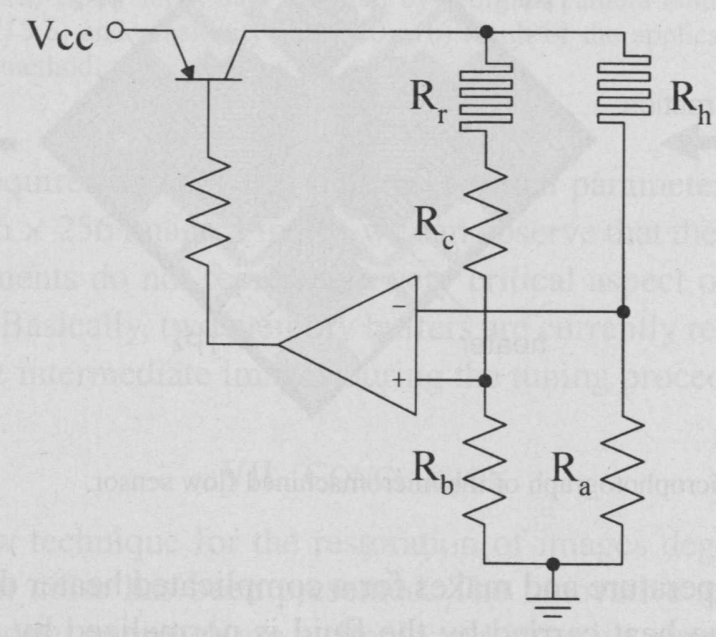

Fig. 3. Thermal bridge for the heater to operate under constant temperature-difference mode.

installed on the ceiling of this duct. The fluid is air and four plates parallel to the flow are used for lamination. Assuming the characteristic length $\mathrm{L}=3.6 \mathrm{~mm}$, the Reynolds' number of the fluid path is about 2,000 at a flow rate of $2,000 \mathrm{l} / \mathrm{h}$. Measurements are carried out at $25^{\circ} \mathrm{C}, 101.325 \mathrm{kPa}$. A float type flow meter using a monolayer film of soap on water surface is used to measure flow rates up to $600 \mathrm{l} / \mathrm{h}$. For higher flow rates up to $12000 \mathrm{l} / \mathrm{h}$, a mass flow meter based on heat transfer by a fluid is used as a reference.

For constant temperature-difference operation, an improved thermal bridge is used to drive the heater. Fig. 3 shows its circuit diagram. In this circuit, $R_{r}$ is a platinum resistor with the same temperature coefficient $\kappa$ as that of the micromachined heater $R_{h}$. Assuming that $R_{r}$ is in thermal equilibrium with the fluid, one can easily derive the following relation:

$$
\Delta \mathrm{T}=\kappa \cdot \mathrm{R}_{\mathrm{c}} \cdot \frac{\mathrm{R}_{\mathrm{a}}}{\mathrm{R}_{\mathrm{b}}}=\kappa \cdot \mathrm{R}_{\mathrm{c}} \cdot \frac{\mathrm{R}_{\mathrm{ho}}}{\mathrm{R}_{\mathrm{r}}}
$$

where $R_{\text {ho }}$ is the heater resistance at $T_{f}$. This relation indicates that $\Delta \mathrm{T}$ can be arbitrarily set by means of $\mathrm{R}_{\mathrm{c}}$.

An instrumentation amplifier is used to take the difference in the electromotive force (EMF) between up- and downstream thermopiles. The EMFs of the two thermopiles placed perpendicular to the flow, on the other hand, are summed to be the monitor output. In the following figures, the difference in the EMF is denoted by V1 and the monitor output by V2.

\section{B. Flow Characteristics}

Fig. 4 shows output voltages of the thermopiles as a function of flow rate. For flow rates up to $100 \mathrm{l} / \mathrm{h}, \mathrm{V} 1$ is proportional to

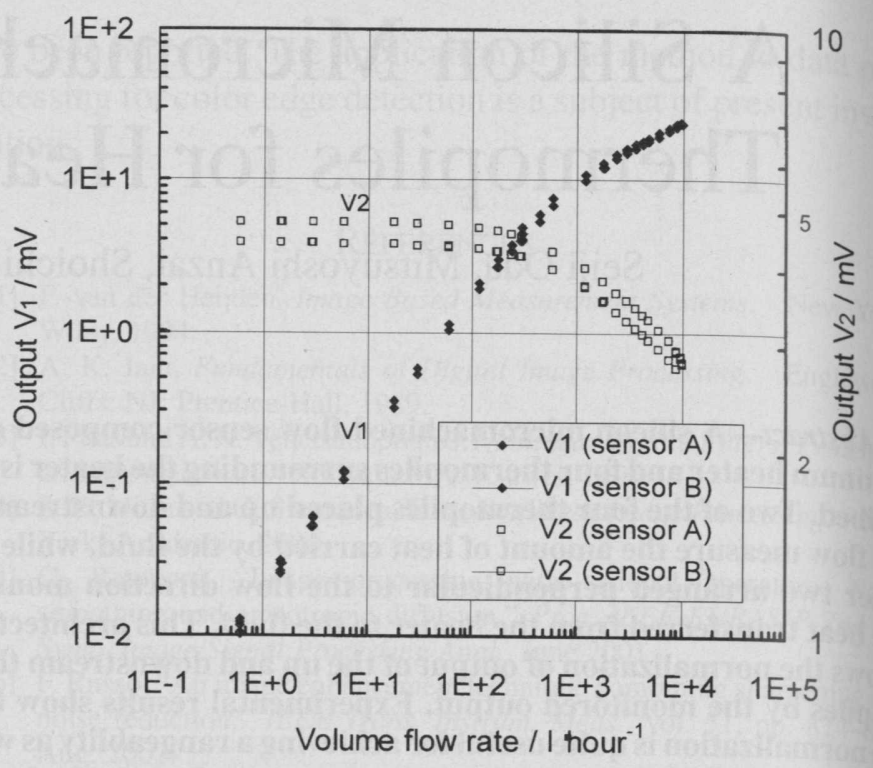

Fig. 4. Output voltages of the thermopiles as a function of flow rate.

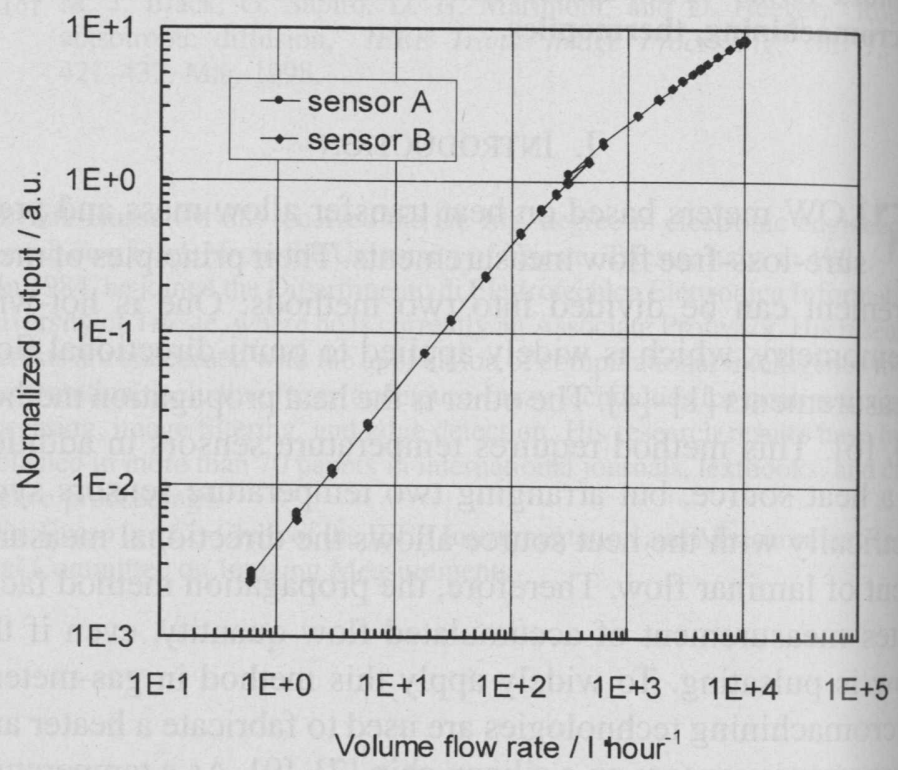

Fig. 5. Normalized outputs as a function of flow rate.

flow rate and V2 remains constant. This indicates that the heat transferred from the heat source to the fluid is independent of fluid velocity and conventional hot-wire anemometry is inapplicable to such a low flow rate measurement [2]. Because of constant heat transfer to the fluid, the same result is also observed in the constant voltage drive of the heater. For flow rates higher than $100 \mathrm{l} / \mathrm{h}, \mathrm{V} 1$ tends to saturate and V2 decreases monotonically with flow rate. This indicates that lower flow rates can be measured by V1 and higher flow rates by V2. Fig. 5 shows the normalized output V1/V2 as a function of flow rate. As can be seen, the rangeability for $\pm 3 \%$ reading error is $1: 1000$, which is much wider than that of a conventional micromachined flow sensor using platinum wires as temperature sensors [8].

Figs. 4 and 5 also compare the performance of two sensors. $\mathrm{A}$ and $\mathrm{B}$, fabricated by the identical process. A discrepancy of about $10 \%$ can be seen in both V1 and V2. This is attributed mainly to the difference in the heater resistances. No appreciable discrepancy can be seen in the normalized output plotted in Fig. 5, however, indicating that the normalization successfully cancels heater dependence. 


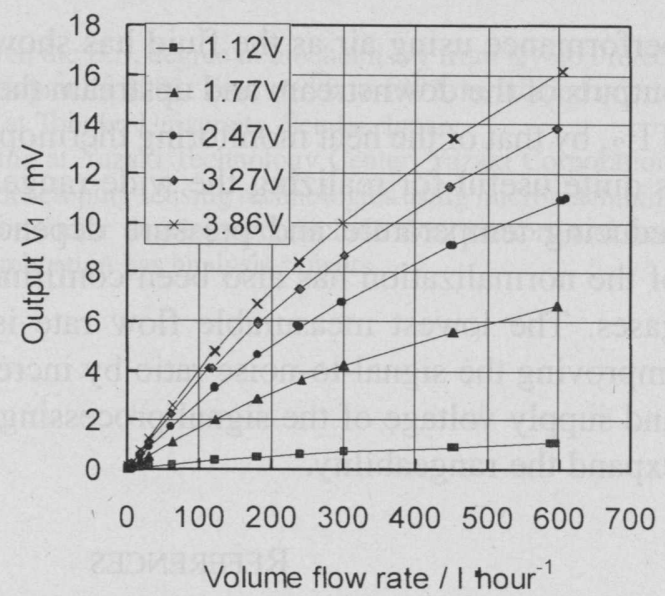

(a)

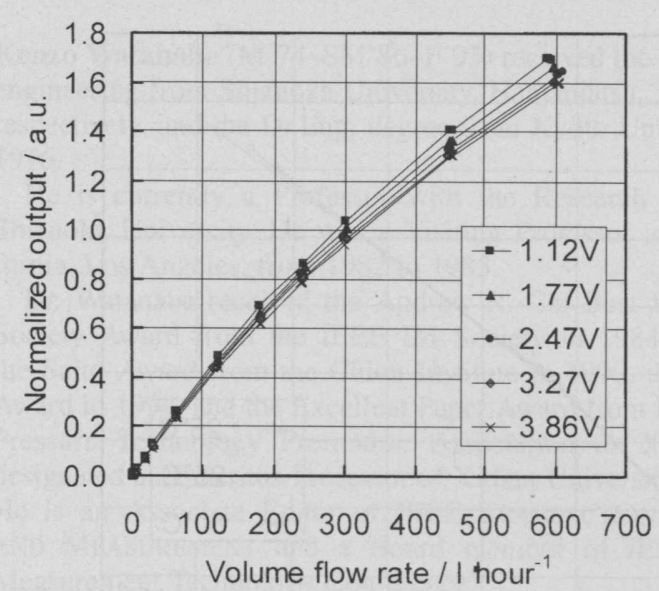

(b)

g. 6. (a) Output voltages and (b) normalized outputs with heater voltage as a parameter.

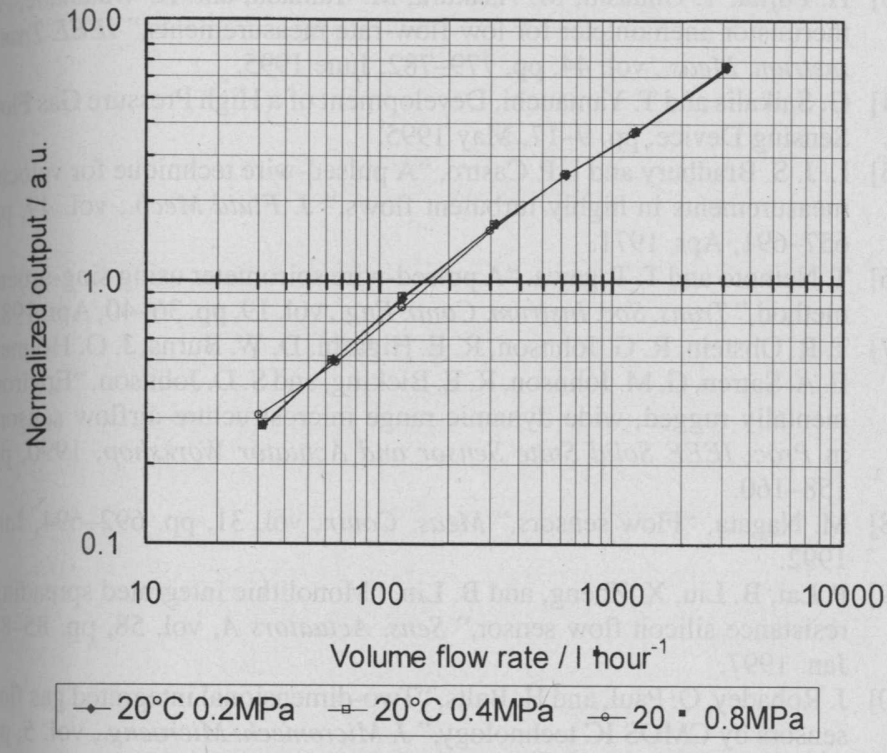

Fig. 7. Pressure dependence of the micromachined flow sensor.

\section{Heater Power Dependence}

Fig. 6 shows the dependence of the flow characteristics on heater voltage. Since the heater is driven in the constant temperature-difference mode, the difference between heater and fluid temperatures increases with heater voltage. Voltage V1 increases almost linearly with heater voltage, as can be seen in Fig. 6(a), but the dependence is greatly reduced by the normalization, as shown in Fig. 6(b). The low sensitivity of the normalized output to heater voltage suggests that constant temperature-difference operation of the heater can be replaced by a much simpler constant voltage drive without temperature dependence deteriorating.

\section{Pressure Dependence}

Fig. 7 shows the dependence of the flow characteristics on pressure. No pressure dependence is observed in the range from atmospheric pressure to $0.8 \mathrm{MPa}$. In these measurements, a $12-\mathrm{mm}$ diameter cylindrical duct is used as the fluid path to withstand high pressure. The same flow meters described earlier are used to measure flow rate.

\section{E. Temperature Dependence}

Fig. 8 shows the dependence on fluid temperature. Temperature dependence still remains in the normalized output. The

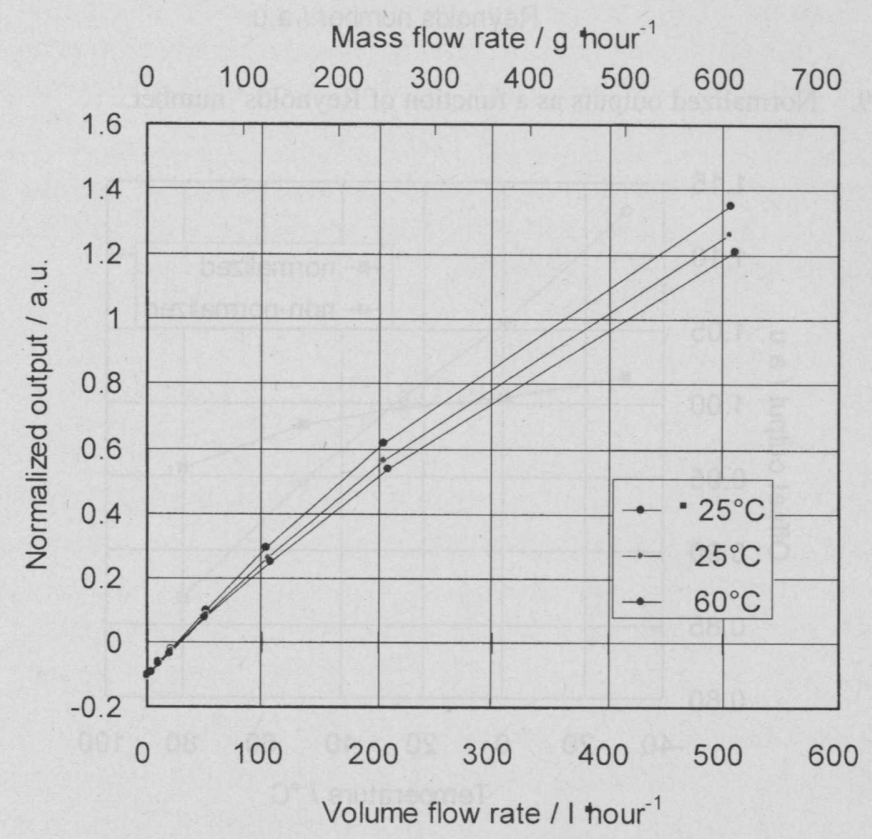

Fig. 8. Temperature dependence of the flow sensor.

measurements were repeated for 100 units, and it was found that all units have the same temperature dependence that can be compensated by a linear function of temperature. The slope of the linear function was found to be approximately $0.2 \% /{ }^{\circ} \mathrm{C}$ in the range from $-25^{\circ} \mathrm{C}$ to $+60^{\circ} \mathrm{C}$.

The EMFs of the thermopiles are independent of pressure, but depend linearly on fluid temperature. The temperature dependence of 100 units was found to be the same despite differences in heater resistances. These experimental facts indicate that temperature dependence is caused solely by thermal properties of the fluid. The effect of the thermal conductivity is canceled by the normalization, as demonstrated in Fig. 5. Therefore, the fluid viscosity is considered to be the main factor responsible for temperature dependence.

To confirm the validity of the above deduction, data in Fig. 8 are replotted in Fig. 9 as a function of the Reynolds' number. The Reynolds number Re is given by

$$
\operatorname{Re}=\rho v \frac{\mathrm{L}}{\eta}
$$

where $\rho$ and $\eta$ are the density and viscosity of the fluid, respectively. The Reynolds' number Re represents mass flow rate $\rho v$ normalized by the viscosity $\eta$. As expected, the normalized 


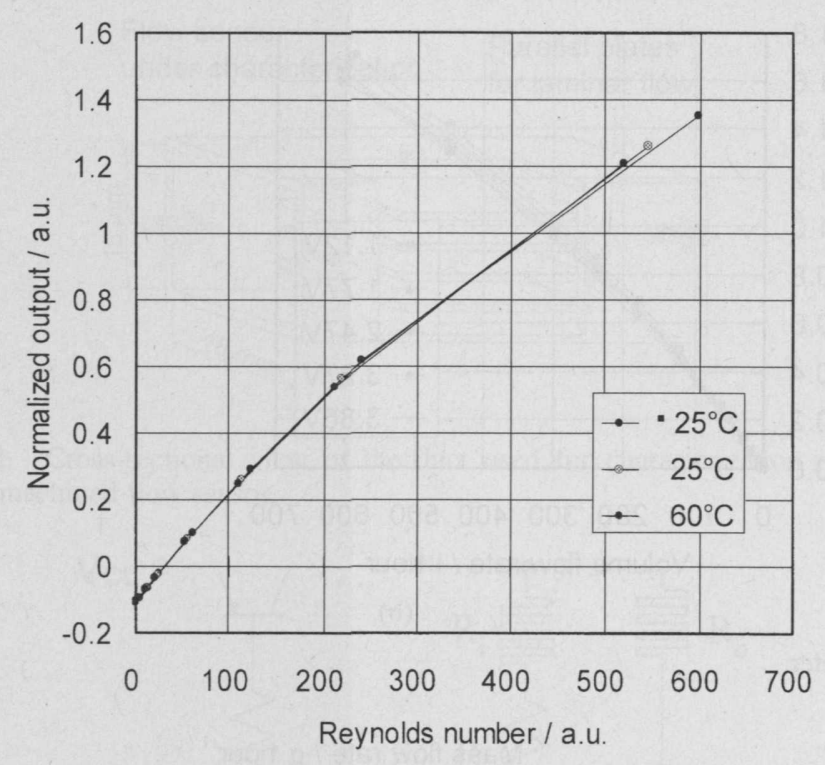

Fig. 9. Normalized outputs as a function of Reynolds' number.

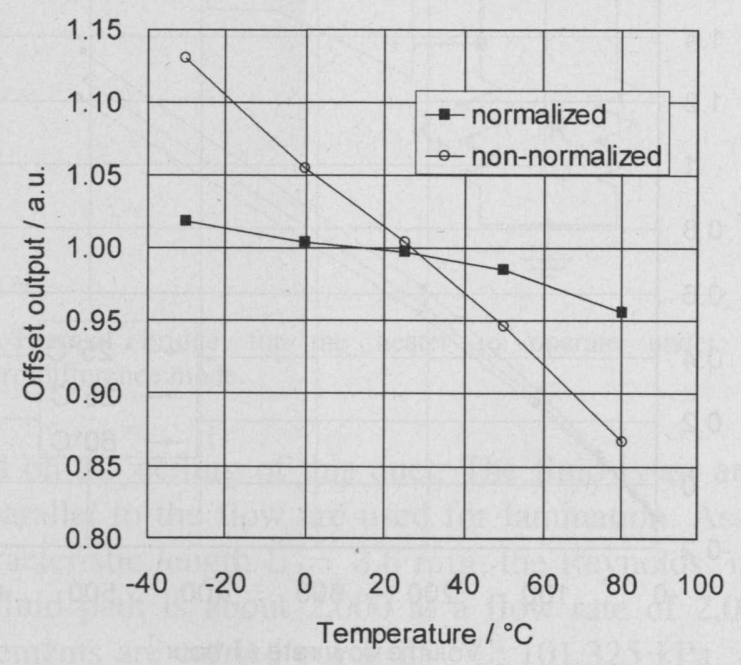

Fig. 10. Temperature dependence of the offset outputs.

output $\mathrm{V}_{1} / \mathrm{V}_{2}$ in Fig. 9 is independent of fluid temperature over the range $-25^{\circ} \mathrm{C}$ to $+60^{\circ} \mathrm{C}$. The viscosity is not a linear function of temperature but proportional to the square root of the absolute temperature. Nevertheless the normalization by the viscosity cancels the linear temperature dependence. This means that the viscosity can be well approximated by a linear function of temperature in the practical temperature range.

\section{F. Offset Drift}

The offset voltage in the EMF depends on the detailed structure of the sensor and also on fluid temperature. Fig. 10 shows a typical temperature dependence of the offset voltage. The ordinate indicates the offset voltage normalized by that at $25^{\circ} \mathrm{C}$. In the figure, temperature dependence of the offset in the normalized V1/V2 output is also plotted. Comparing the two plots, one notices that the normalization is also useful for reducing temperature dependence, thereby resulting in more accurate measurements of low flow rates.

\section{CONCLUSION}

A silicon micromachined flow sensor composed of a platinum heater and four thermopiles was described. Evaluated performance using air as the fluid has shown that normalizing outputs of the downstream and upstream thermopiles, $\mathrm{TP}_{1}$ and $\mathrm{TP}_{2}$, by that of the heat monitoring thermopiles, $\mathrm{TP}_{3}$ and $\mathrm{TP}_{4}$ is quite useful for realizing the wide rangeability and also for reducing temperature and pressure dependence. The validity of the normalization has also been confirmed for hydrocarbon gases. The lowest measurable flow rate is limited by noise. Improving the signal-to-noise ratio by increasing heater power and supply voltage of the signal processing circuit will further expand the rangeability.

\section{REFERENCES}

[1] A. B. Bauer, "Direct measurement of velocity by hotwire anemometry," AIAA J., vol. 3, pp. 1189-1191, June 1965.

[2] A. E. Perry, Hot-Wire Anemometry. Oxford, U.K.: Clarendon, 1982

[3] H. Fujita, T. Ohhashi, M. Asakura, M. Yamada, and K. Watanabe, "A thermistor anemometer for low-flow-rate measurements," IEEE Trans. Instrum. Meas., vol. 44, pp. 779-782, June 1995.

[4] G. Saikalis and T. Yamauchi, Development of a High Pressure Gas Flow Sensing Device, pp. 9-17, May 1995.

[5] L. J. S. Bradbury and I. P. Castro, "A pulsed-wire technique for velocity measurements in highly turbulent flows," J. Fluid Mech., vol. 49, pp. 657-691, Apr. 1971.

[6] T. Nemoto and T. Togawa, "A pulsed-wire spirometer using sing-around method," Trans. Soc. Instrum. Contr. Eng., vol. 19, pp. 36-40, Apr. 1983.

[7] T. R. Ohstein, R. G. Johnson, R. E. Higashi, D. W. Burns, J. O. Holmen, E. A. Satren, G. M. Johnson, R. E. Bicking, and S. D. Johnson, "Environmentally rugged, wide dynamic range microstructure airflow sensor," in Proc. IEEE Solid State Sensor and Actuator Workshop, 1990, pp. $158-160$.

[8] M. Nagata, "Flow sensors," Meas. Contr., vol. 31, pp. 692-694, June 1992.

[9] P. Lai, B. Liu, X. Zheng, and B. Lin, "Monolithic integrated spreadingresistance silicon flow sensor," Sens. Actuators A, vol. 58, pp. 85-88, Jan. 1997.

[10] J. Robadey, O. Paul, and H. Balts, "Two-dimensional integrated gas flow sensors by CMOS IC technology," J. Micromech. Microeng., vol. 5, pp. 243-250, Ma. 1995

[11] G. Kaltsas and A. G. Nassiopoulou, "Novel CMOS compatible monolithic gas flow sensor with porous silicon thermal isolation," Sens. Actuators A, vol. 76, pp. 133-138, 1999.

[12] R. E. Oosterbroek, T. S. J. Lammerink, J. W. Berenschot, G. J. M. Krijnen, M. C. Elwenspock, and A. van der Berg, "A micromachined pressure/flow-sensor," Sens. Actuators A, vol. 77, pp. 167-177, 1999.

[13] K. A. A. Makinwa and J. H. Huijing, "Constant power operation of a two-dimensional flow sensor using thermal sigma-delta modulation techniques," in Proc. IEEE Instrumentation and Measurement Technology Conf., 2001, pp. 1577-1580.

[14] M. Nakamura, S. Uematsu, S. Oda, and M. Yamaura, "Instantaneous gas flowmeter using thermopile type micro flow-rate sensor," in Proc. Fluid Meas. Contr. Symp., Nov. 2000, pp. 21-24.

[15] S. Oda, Y. Okamoto, O. Kimura, M. Anzai, S. Uematsu, M. Seto, K. Kobayashi, K. Tashiro, and K. Nukui, "Flow sensor with thermopiles," in Proc. Fluid Meas. Contr. Symp., Nov. 2001, pp. 37-40.

Seiji Oda received the B.S. degree in applied physics from the Science University of Tokyo, Tokyo, Japan, in 1986.

He worked on collaborative research of ceramics, optical crystals, and laser systems at the Tokyo Research Center, Tosoh Corporation, and the Institute of Physical and Chemical Research until 1993. He is currently working on application, evaluation, and theoretical analysis of micromachined flow sensors at Yazaki Technology Center, Yazaki Corporation, Shizuoka, Japan.

Mitsuyoshi Anzai received the B.S. degree in electronic engineering from the Tokyo Denki University, Tokyo, Japan, in 1985.

He is currently with Yazaki Technology Center, Yazaki Corporation, where he is engaged in research and development of electronic circuits to drive a variety of micromachined sensors. 
choichi Uematsu received the B.S. degree in biochemistry from Kyoto Prefecghoul University, Kyoto, Japan, in 1983. From 1990 to 1992, he studied microchining technologies at Tohoku University, Sendai, Japan.

Currently, he is working at Yazaki Technology Center, Yazaki Corporation, where he is in charge of developing sensing technologies using micromachining whologies. The sensors under development include flow sensors and heat gchnsfer and catalytic combustion gas analysis sensors.
Kenzo Watanabe (M'74-SM'86-F'93) received the B.E. and M.E. degrees in engineering from Shizuoka University, Hamamatsu, Japan, in 1962 and 1966, respectively, and the Dr.Eng. degree from Kyoto University, Kyoto, Japan, in 1976.

$\mathrm{He}$ is currently a Professor with the Research Institute of Electronics, Shizuoka University. He was a Visiting Professor at the University of California, Los Angeles, from 1982 to 1983.

Dr. Watanabe received the Andrew R. Chi Best Paper Award and the IM Society Award from the IEEE IM Society in 1984 and 1999, respectively, the Saito Award from the Chion Institute in 1990, the Takayanagi Memorial Award in 1994, and the Excellent Paper Award from Hydraulic and Pneumatic Pressure Technology Promotion Association in 2002. He was also been designated an Emeritus Professor of Xidian University, Xi' an, China, in 1997. $\mathrm{He}$ is an Associate Editor of IEEE TRANSACTIONS ON INSTRUMENTATION AND MEASUREMENT and a Board member of IEEE Instrumentation and Measurement Technology Conference. 